\title{
Effectiveness of companion-intensive
} multi-aspect weight management in Chinese adults with obesity: a 6-month multicenter randomized clinical trial

\author{
Wanzi Jiang ${ }^{1 \dagger}$, Shushu Huang ${ }^{1,2+}$, Shuai Ma ${ }^{1 \dagger}$, Yingyun Gong ${ }^{1}$, Zhenzhen Fu' ${ }^{1}$ Li Zhou ${ }^{3}$, Wen Hu${ }^{4}$, \\ Guofang Mao ${ }^{5}$, Zhimin Ma ${ }^{5}$, Ling Yang ${ }^{6}$, Guangfeng Tang ${ }^{7}$, Xiaofang Sun ${ }^{8}$, Ping Zhang ${ }^{9}$, Jianling Bai ${ }^{10}$, \\ Lei Chen ${ }^{11 \dagger}$, Bimin Shi ${ }^{12^{*}}$, Xinhua Ye ${ }^{13^{*}}$ and Hongwen Zhou ${ }^{1 *}$ (D)
}

\begin{abstract}
Background: Obesity is a globally increasing health epidemic requiring early lifestyle intervention. Our main objective was to examine the effectiveness of companion-intensive multi-aspect weight management (CIMWM) in Chinese adults with obesity.

Methods: In this 6-month, prospective, open-label, multicenter, randomized controlled clinical trial, we recruited 272 obese adults aged $18-50$ years with a body mass index $(B M I) \geq 28.0 \mathrm{~kg} / \mathrm{m}^{2}$ and capable of using smartphones. CIMWM $(n=136)$ offered both daily online instructions and monthly face-to-face guidance by physicians, dietitians, and health managers along with the provision of meal replacements in the first 3 months. Traditional multi-aspect weight management (TMWM, $n=136$ ) provided monthly face-to-face guidance by the same panel of professionals and the same meal replacements as CIMWM group, but required subjects to complete daily self-monitoring instead of offering daily online instructions. Body composition and metabolic parameters were assessed at baseline, 1, 2, 3, and 6 months by physicians. The primary outcomes were clinically-significant weight loss and changes in BMI and body composition.
\end{abstract}

Results: Participants in both groups showed significantly reduced BMI, body fat mass (BFM), visceral fat area (VFA), and HOMA-IR $(p<0.05)$. CIMWM was shown to be superior to TMWM in the improvement of clinicallysignificant weight loss, BMI, total cholesterol (TC), the body composition parameters BFM and the skeletal muscle

\footnotetext{
*Correspondence: shibimin@163.com; Endocrine1314@163.com;

drhongwenzhou@njmu.edu.cn

tWanzi Jiang, Shushu Huang, Shuai Ma, and Lei Chen have contributed

equally to this work

${ }^{1}$ Department of Endocrinology and Metabolism, The First Affiliated

Hospital of Nanjing Medical University, Jiangsu Province Hospital,

Nanjing 210029, China

12 Department of Endocrinology, The First Affiliated Hospital of Soochow

University, Suzhou 215000, China

${ }^{13}$ Department of Endocrinology, The Affiliated Changzhou No. 2 People's

Hospital of Nanjing Medical University, Changzhou 213000, China

Full list of author information is available at the end of the article
}

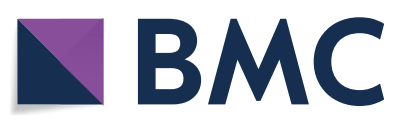

(c) The Author(s) 2021, corrected publication 2021. Open Access This article is licensed under a Creative Commons Attribution 4.0 International License, which permits use, sharing, adaptation, distribution and reproduction in any medium or format, as long as you give appropriate credit to the original author(s) and the source, provide a link to the Creative Commons licence, and indicate if changes were made. The images or other third party material in this article are included in the article's Creative Commons licence, unless indicated otherwise in a credit line to the material. If material is not included in the article's Creative Commons licence and your intended use is not permitted by statutory regulation or exceeds the permitted use, you will need to obtain permission directly from the copyright holder. To view a copy of this licence, visit http://creativecommons.org/licenses/by/4.0/. The Creative Commons Public Domain Dedication waiver (http://creativecommons.org/publicdomain/zero/1.0/) applies to the data made available in this article, unless otherwise stated in a credit line to the data. 
mass-to-visceral fat area ratio (SN) $(p<0.05)$. The non-alcoholic fatty liver disease score (NFS) was negatively related to $S / N$ at baseline. After weight management, NFS was lowered among individuals with levels in the highest tertile $(p<0.05)$. Metabolic memory in terms of the continuous reduction of BMI, BFM, and TC was retained up to 6 months in spite of participants transferring to self-monitoring assessment in the final 3 months.

Conclusions: The CIMWM strategy in obese Chinese adults is proved to be more effective than TMWM in weight loss, and motivates greater adherence to intervention and lifestyle reprogramming.

Trial registration Chinese Clinical Trial Registry, ChiCTR1800017463, Registered July 31, 2018. http://www.chictr.org.cn/ showproj.aspx?.proj=29649.

Keywords: Companion-intensive multi-aspect lifestyle intervention, Obesity, Weight loss, Body composition

\section{Background}

Obesity is a global health epidemic. According to the World Health Organization (WHO), up to $57.8 \%$ of adults worldwide will be classified as obese by 2030 [1]. The prevalence of obesity among Chinese adults is $\sim 11 \%$ [2]. Body mass index (BMI) is a widely used parameter to define overweight and obese status in adults [3]. In China, individuals within a BMI range of $24.0-27.9 \mathrm{~kg} / \mathrm{m}^{2}$ are defined as overweight and those with BMI $\geq 28.0 \mathrm{~kg} / \mathrm{m}^{2}$ as obese [4].

Obesity is an underlying risk factor for a series of disorders, such as diabetes, non-alcoholic fatty liver disease (NAFLD), coronary heart disease, sleep apnea syndrome, stroke, and cancer, which are associated with significantly increased morbidity and mortality [5, 6]. Effective treatment options and prevention strategies to control obesity are therefore a top priority for healthcare systems [7]. Scientifically sound and appropriate weight loss treatments are essential to lower the risk of obesity-related diseases. Weight loss treatments that have been proven effective for patients with obesity include lifestyle modification, conventional pharmacologic treatments and surgical treatments [8]. In particular, lifestyle modification is the foundation of weight loss and should be consistently implemented [9].

Intensive lifestyle modification involves alterations in five indispensable aspects: diet, exercise, psychotherapy, behavioral intervention, and health education. The Diabetes Remission Clinical Trial (DiRECT) in the UK showed significant benefits in patients with type 2 diabetes mellitus (T2DM) and obesity who underwent a primary care-led weight management program, with almost half of the participants achieving remission to a non-diabetic state and requiring no further treatment by 12 months [10]. Another study demonstrated that a Web-Based Behavior Change Program could help achieve weight loss and reduce cardiovascular disease (CVD) risk in obese individuals, supporting the internet as a viable medium for weight loss management [7]. Records showed that in 2014, 92\% of the population in China had access to mobile phones, with smartphones being widely used by young and middle-aged individuals [11].

Although early lifestyle intervention is important for the control of obesity, it is difficult for patients to adhere to daily modifications on their own, so that intensive supervision by doctors or other professionals is necessary for successful weight management. Irregular face-to-face guidance by the community and hospitals is the traditional method used for patients with obesity in China, which often results in poor achievement of self-monitoring ability and weight loss. To resolve this issue, we proposed the implementation of a Companion-Intensive Multi-aspect Weight Management (CIMWM) strategy focusing on a combination of online and offline medical interventions with daily lifestyle supervision and guidance of diet and exercise. To evaluate this strategy, we conducted a multicenter, randomized, controlled clinical intervention trial for adults with obesity in China from 2018 to 2019, which aimed to compare the efficacy and feasibility of two different medical weight management intervention programs. CIMWM offers both daily online instructions and monthly face-to-face guidance by physicians, dietitians and health managers, along with provision of meal replacements in the first 3 months. Individuals of traditional multi-aspect weight management (TMWM) were provided with monthly face-to-face guidance by the same panel of professionals and the same meal replacements as CIMWM group, but were required to complete daily self-monitoring instead of being offered with daily online instructions. Changes in clinical indicators, such as lipid profiles, blood glucose, Homeostatic Model Assessment of Insulin Resistance (HOMA-IR), blood pressure, and body composition, were additionally evaluated. This study is beneficial in raising public awareness of obesity prevention, proposing the appropriate obesity intervention programs, and providing evidence for relevant guidelines and formulation of local government health policies [12]. 


\section{Methods}

\section{Study design}

This study was designed as a prospective, open, multicenter, 6-month, randomized, controlled clinical trial, registered at the Chinese Clinical Trial Registry (ChiCTR1800017463), and approved by the Ethics Committee at the First Affiliated Hospital of Nanjing Medical University (Nanjing, Jiangsu, China) (2018-SR-069). The recruitment period was from August 1, 2018 to June 31, 2019. Recruitment strategies included advertisements in social media, e.g. WeChat, along with posters and referrals from the outpatient clinic. Written informed consents were obtained from all participants.

\section{Participants}

This clinical trial was conducted primarily in 11 tertiary care hospitals across China, including (1) 86 patients from the First Affiliated Hospital of Nanjing Medical University (Jiangsu Province Hospital, Primary center), (2) 20 patients from the Affiliated Changzhou No. 2 People's Hospital of Nanjing Medical University, with 4 withdrawn, (3) 28 patients from the First Affiliated Hospital of Soochow University, with 3 withdrawn, (4) 20 patients from Suzhou Municipal Hospital Affiliated to Nanjing Medical University, with 1 withdrawn, (5) 10 patients from the Affiliated Suzhou Science \&Technology Town Hospital of Nanjing Medical University, with 1 withdrawn, (6) 19 patients from the Affiliated Hospital of Jiangsu University, (7) 13 patients from the Affiliated Huaian No.1 People's Hospital of Nanjing Medical University, (8) 27 patients from the Second People's Hospital of Huai'an, the Affiliated Huai'an Hospital of Xuzhou Medical University, (9) 15 patients from Northern Jiangsu People's Hospital, (10) 25 patients from the First People's Hospital of Chuzhou, with 9 withdrawn, and (11) 9 patients from the Second Affiliated Hospital of Dalian Medical University.

The duration of the study was from July 1, 2018 to December 30, 2019.

\section{Inclusion and exclusion criteria}

Eligible adults with obesity aged between 18 and 50 years with $\mathrm{BMI} \geq 28.0 \mathrm{~kg} / \mathrm{m}^{2}$, capable of using smartphones and operating the mobile application "Medical Weight Management" (Guangzhou ND-fit Nutrition and Health Consulting Co. Ltd) were included. Participants additionally needed to follow the guidance of dietitians and health managers, and monitor relevant indicators in accordance with the program requirements throughout the study. The complete inclusion and exclusion criteria are presented in Fig. 1.

\section{Intervention}

A multi-aspect team comprising physicians, dietitians, and health managers delivered the program over a 6-month period. Participants in the CIMWM group were provided with two Fit Nutrition Bars (Guangzhou ND-fit Nutrition and Health Consulting Co. Ltd) per day in the first 3 months as well as monthly face-to-face guidance and daily online instructions via the mobile application "Medical Weight Management", which allowed uploading data of daily weight and food diaries. This information helped our team of experts to manage and guide the subjects online in real time. The TMWM group underwent a separate program with monthly face-to-face guidance by the same multi-aspect team and the same meal replacements, but with daily selfmonitoring instead of a real-time guidance via mobile application. In the final 3 months, all participants were transferred to the self-monitoring period of the program. All participants were followed up at 1, 2, 3, and 6 months after randomization for assessment. Details of the interventions are presented in Fig. 1.

Features of the diet and exercise intervention programs are described below. Based on age, sex, standard body weight, typical caloric intake, and physical activities, participants received an individualized calorie-restricted diet (CRD) [13] plan with 12001800 kilocalories (kcal) per day, which was developed by registered dietitians, providing $40-55 \%$ of daily calories from carbohydrates, $20-30 \%$ from fat, and $15-20 \%$ from protein. Fit Nutrition Bars consisting of whey protein, soy protein isolate, chia seeds, oligosaccharides, collagen, and konjac extract rich in dietary fiber and $\gamma$-aminobutyric acid were provided as meal replacements for participants from both groups in the first 3 months. A single bar weighs $30 \mathrm{~g}$ and provides $111 \mathrm{kcal}$ with $9.15 \mathrm{~g}$ protein, $1.8 \mathrm{~g}$ fat, $12.48 \mathrm{~g}$ carbohydrate, and $3.93 \mathrm{~g}$ dietary fiber. Both groups were additionally provided with calcium, vitamin $\mathrm{D}$, and multivitamin mineral tablets.

Individualized exercise plans were created by health managers for each participant based on their health status and exercise capacity. The exercise plan included a weekly 160 min group exercise session. Over 6 months, participants were instructed to exercise for $40 \mathrm{~min} /$ day, 4 days a week, starting with 5 min warm-up exercises, followed by $10 \mathrm{~min}$ and $30 \mathrm{~min}$ of resistance and aerobic exercises, respectively, and ending with 5 min muscle stretching exercises.

\section{Assessments}

Assessments were conducted face-to-face at baseline, 1, 2,3 , and 6 months by physicians. 


\section{CIMWM group:}

a.daily online instruction monitoring by physicians, dietitians and health

managers on calorie-restricted diet with standardized meal replacements and individualized exercise

b.monthly face-to-face guidance by physicians, dietitians and health managers TMWM group:

c. daily self-monitoring on calorie-restricted diet with standardized meal replacements and individualized exercise

b. monthly face-to-face guidance by physicians, dietitians and health managers

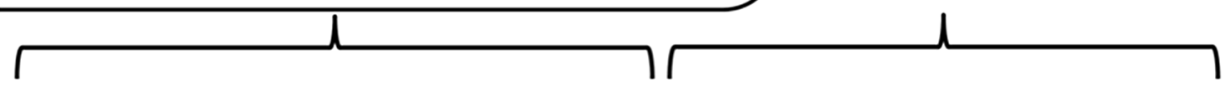

\section{-2 weeks 0 month 1 month 2 month 3 month

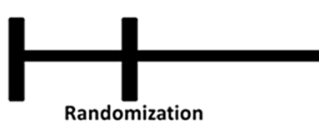

Visit 1 Visit 2

Visit 3

Visit 4

Visit 5

Visit 6

Visit 1:Baseline assessment Physical examination: height, body weight, blood pressure, heart rate, ECG

Hematology test:

OGTT, blood lipid profile, blood routine,

liver and kidney function, $\mathrm{HbA1C}$,

Electrolyte, thyroid function, ACTH, cortisol

Urine test:

urine routine, urine pregnancy test (female)
Visit 2, 3, 4, 5, 6:

Anthropometry:

height, body weight, waist and hip circumference, blood pressure, body composition

\section{Visit 2, 5, 6:}

Hematology test:

OGTT, blood lipid profile, liver and kidney function, $\mathrm{HbA1C}$, blood routine

Urine test:

urine routine, urine pregnancy test (female)

The following patients were included:

Eligible obese adults aged between 18 and 50 years with a BMI $\geq 28.0 \mathrm{~kg} / \mathrm{m} 2$ and capable of using smartphones and operating the mobile application "Medical Weight Management" were included. Participants also needed to follow the guidance of dietitians and health managers, and monitor and record relevant indicators in accordance with the program requirements throughout the study.

The following patients were excluded :

(i) those with gastrointestinal diseases accompanied by malabsorption;

(ii) those with active liver diseases or obvious liver function damage, defined by alanine aminotransferase (ALT) levels $>2.5$ times the normal upper limit;

(iii) those with a history of unstable or rapidly progressive renal disease or renal impairment, defined by serum creatinine $\geq 133 \mu \mathrm{mol} / \mathrm{L}$ in male subjects or $\geq 125 \mu \mathrm{mol} / \mathrm{L}(1.4 \mathrm{mg} / \mathrm{dL}$ ) in female subjects;

(iv) those diagnosed with diabetes or obesity caused by Cushing syndrome and other endocrinological disorders;

(v) those who had taken the following drugs within the last 4 weeks-lipid-lowering drugs such as statins and fibrates, anti-obesity drugs, hepatoprotective drugs, glucocorticoids, and drugs that may affect blood glucose and blood lipids;

(vi) those who had undergone weight-reduction surgery;

(vii) those with mental disorders, unwillingness to communicate with others, or having a language barrier, leading to insufficient understanding of the research program or incapability to cooperate with researchers;

(viii) those with severe cardiopulmonary insufficiency, hypothyroidism, or malignant tumors;

(ix) pregnant women, breastfeeding women, those planning a pregnancy during the course of the study, and reproductive-aged women but refusing to use effective, medically approved contraception during the study;

$(x)$ those with a history of alcoholism (quantity of alcohol consumed by men $\geq 140 \mathrm{~g}$ per week, and that consumed by women, $\geq 70$ g per week);

(xi) those with physical dysfunction, affecting aerobic exercise and resistance exercise;

(xii) those currently participating in other weight-loss programs or who had lost more than $5 \%$ of their body weight in the past 6 months;

(xiii) those with poorly controlled severe hypertension, i.e., systolic blood pressure $\geq 180 \mathrm{mmHg}$ and/or

diastolic blood pressure $\geq 110 \mathrm{mmHg}$ and those with systolic blood pressure $<95 \mathrm{mmHg}$.

Fig. 1 Structure of the weight management programs 


\section{Primary outcomes}

Clinically-significant weight loss (defined as weight loss $\geq 5 \%$ ) in different weight managements groups was one of the primary outcomes. Body mass index (BMI), body weight (BW), body fat percentage (BFP), body fat mass (BFM), fat-free mass (FFM), skeletal muscle mass (SMM), and visceral fat area (VFA) were measured in participants while standing on an automated hand-tofoot bioelectrical impendence device (JAWON IOI353 Body composition analyzer, Korea) with bare feet and light clothing.

\section{Secondary outcomes}

Anthropometric measurements, such as height, waist circumference (WC) and hip circumference (HC) accurate to the nearest $0.01 \mathrm{~cm}$, were obtained using standard techniques. Blood pressure (while seated) was recorded as the average of two measures. Blood samples were obtained in the morning after an overnight fast. The BECKMAN-AU5800 automatic biochemical analyzer was used for determination of fasting plasma glucose (FPG), alanine aminotransferase (ALT), aspartate aminotransferase (AST), total cholesterol (TC), triglyceride (TG), high-density lipoprotein cholesterol (HDL-C), lowdensity lipoprotein cholesterol (LDL-C), lipoprotein(a) $[\mathrm{Lp}(\mathrm{a})]$, creatinine $(\mathrm{Cr})$, uric acid (UA), platelet (PLT), and albumin (ALB) levels. Fasting insulin (FINS) and fasting $\mathrm{C}$ peptide (FCP) contents were determined via chemiluminescence, and glycated hemoglobin (HbA1c) was detected using high-performance liquid chromatography (Bole, USA). The non-alcoholic fatty liver disease score (NFS) was calculated as follows:

- $\quad 1.675+0.037 \times$ age $\quad$ (years $)+0.094 \times$ BMI $\quad(\mathrm{kg} /$ $\left.\mathrm{m}^{2}\right)+1.13 \times$ impaired fasting glucose/diabetes (yes $=1$, no $=0)+0.99 \times \mathrm{AST} / \mathrm{ALT}$ ratio $-0.013 \times$ platelet count $\left(\times 10^{9} / \mathrm{L}\right)-0.66 \times$ albumin $(\mathrm{g} / \mathrm{dL})[14]$.

Advanced fibrosis was accurately excluded by applying the NFS low cut-off point $(-1.455)$ while its presence was diagnosed with high accuracy by applying the NFS high cut-off point (0.676) [14]. HOMA-IR was calculated using the formula: FPG $(\mathrm{mmol} / \mathrm{L}) \times$ FINS $(\mathrm{mIU} / \mathrm{L}) / 22.5$.

Diabetes mellitus was defined as either FPG $\geq 7 \mathrm{mmol} / \mathrm{L}$ or $2 \mathrm{~h}$ oral glucose tolerance test (OGTT) blood glucose $(2 \mathrm{hBG}) \geq 11.1 \mathrm{mmol} / \mathrm{L}$, and impaired fasting glucose (IFG) was defined as $6.1 \mathrm{mmol} / \mathrm{L} \leq \mathrm{FPG}<7 \mathrm{mmol} / \mathrm{L}$ and $2 \mathrm{hBG}<7.8 \mathrm{mmol} / \mathrm{L}[15]$.

\section{Statistical analysis}

This study was powered using two previous technologybased weight management studies [16, 17], based on the percentage of individuals with clinically-significant weight loss (defined as weight loss $\geq 5 \%$ ) in groups using different weight managements. A sample size of 214 subjects, 107 in each arm, is sufficient to detect a clinically important difference of about $20 \%$ between groups using a two-tailed z-test of proportions with $90 \%$ power and a 5\% level of significance. Considering a dropout rate of $20 \%$, the sample size required is 272 (136 per group). Accordingly, competitive enrollment was initiated at each branch center. Enrolled patients were numbered in adjacent sequences using the randomized block design (block size $=4$ ). Random assignment codes were generated by statistics professionals using the SAS 9.2 software proc plan program according to a 1:1 allocation ratio [7].

Analyses were performed using SPSS for Macintosh version 25.0 (SPSS Inc, Chicago, IL, USA) and SAS 9.2 software proc plan program. Demographic and baseline characteristics were evaluated with the aid of descriptive statistics. Data are represented as mean $\pm S D$ for continuous variables and as percentages for categorical variables. Data sets involving the baseline, 1-month, 2-month, 3-month, and 6-month index of the participants in each group were assessed using repeated measurement ANOVA. Spearman's rank correlation was performed to evaluate the relationships between the changes of physical examination and hematology indices at the 6-month point. To compare the characteristics of continuous variables between the two groups, the differences of changes from baseline to $1,2,3$, and 6 months between two groups were assessed with mixed model controlling for baseline index using SAS 9.2. For categorical variables, the chi-square test was used. Two-sided $p$ values $<0.05$ were considered statistically significant.

\section{Results}

Participants

Participants were recruited from August 2018 to June 2019 (Fig. 2). Among the 378 eligible subjects, 272 (72\%) were included and randomly assigned to study groups at a 1:1 allocation ratio. Overall, 254 (93.3\%) participants completed the 6-month weight management intervention study.

\section{Anthropometric, metabolic, and clinical characteristics of the participants in each group before and after the weight loss intervention}

The baseline characteristics of both groups are presented in Table 1. No significant intergroup differences were observed except for FCP.

The clinical and demographic characteristics of the participants in each group are presented in Additional file 1: Table S1. Compared to baseline, BMI in both groups was significantly decreased at each visit point ( $p<0.001$, Fig. 3a). Loss of body weight, including BFM and SMM was sustained during the whole 6 months in both groups when comparing with baseline 


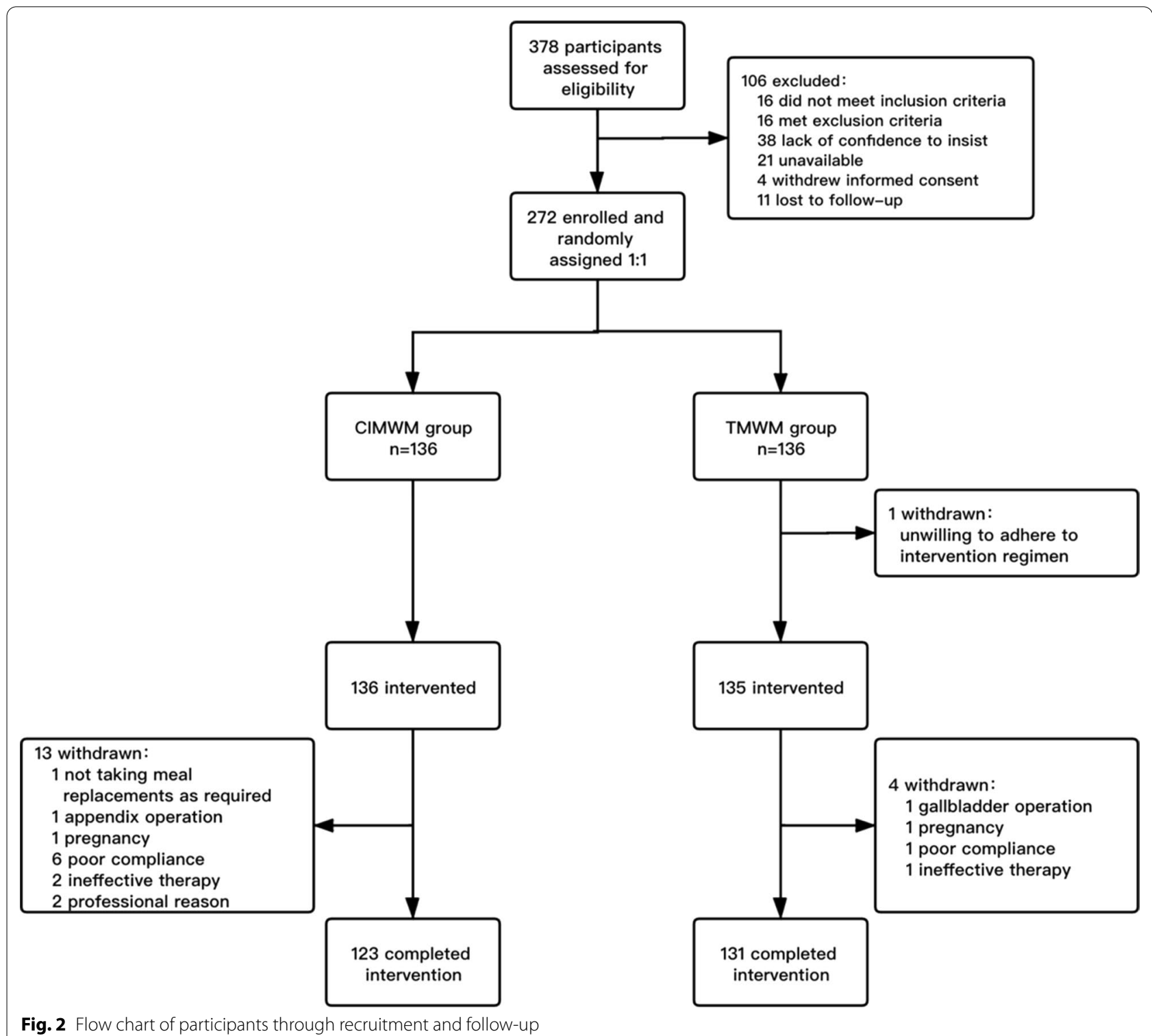

$(p<0.001$, Additional file 1: Table S1, Fig. 3g). Additionally, BFP, BFM, VFA, WC, HC, SBP, DBP, ALT, TC, TG, LDL-C, FINS, and HOMA-IR, were significantly decreased at both 3 and 6 months $(p<0.05$, Additional file 11 Table S1, Fig. 3b, d, e, f). Nevertheless, at 6 months compared with baseline, FFM was slightly decreased by $2.86 \mathrm{~kg}$ in CIMWM group $(p<0.001)$ and $2.05 \mathrm{~kg}$ in TMWM group $(p<0.001)$, while SMM was decreased by $2.06 \mathrm{~kg}$ in CIMWM group $(p<0.001)$ and $1.48 \mathrm{~kg}$ in TMWM group $(p<0.001)$. However, sustaining increase of $\mathrm{S} / \mathrm{V}$ over the 6 months was only observed in the CIMWM group, compared with baseline $(p<0.001$, Fig. 3c). Additionally, only in the CIMWM group, the number of subjects with IFG was significantly decreased at both 3 and 6 months. Moreover, only at 6 months, UA was markedly declined from baseline in both groups. HDL-C levels was gradually increased from baseline to the end of the study period in CIMWM group $(p<0.001)$.

The correlation coefficient heatmap (Fig. 4) indicated that the variations in BMI and BFM from baseline to 6 months were positively correlated with those of ALT, AST, TC, TG, FPG, FINS, FCP, HbA1c, HOMA-IR, SBP, and DBP (all $p<0.05$ ) and inversely correlated with HDL-C changes $(p<0.05)$. Positive associations were observed between 6-month changes of VFA and ALT, TC, TG, FPG, FCP, HbA1c, SBP, as well as DBP values $(p<0.05)$. Simultaneously, changes in $\mathrm{S} / \mathrm{V}$ were positively 
Table 1 Baseline characteristics of participants in the CIMWM and TMWM groups ${ }^{\mathrm{a}}$

\begin{tabular}{|c|c|c|c|}
\hline & CIMWM & TMWM & $p$ value $^{b}$ \\
\hline Age (years) & $31.52 \pm 6.43$ & $32.16 \pm 6.39$ & 0.427 \\
\hline $\operatorname{Sex}(M / F)$ & $72 / 51$ & $77 / 54$ & 0.969 \\
\hline $\mathrm{BMI}\left(\mathrm{kg} / \mathrm{m}^{2}\right)$ & $32.36 \pm 3.40$ & $32.48 \pm 3.59$ & 0.800 \\
\hline $\mathrm{BW}(\mathrm{kg})$ & $92.77 \pm 14.91$ & $92.98 \pm 14.08$ & 0.909 \\
\hline BFP (\%) & $34.60 \pm 4.54$ & $35.03 \pm 4.72$ & 0.459 \\
\hline BFM (kg) & $32.03 \pm 6.32$ & $32.61 \pm 7.06$ & 0.490 \\
\hline FFM (kg) & $60.82 \pm 11.14$ & $60.30 \pm 9.88$ & 0.689 \\
\hline SMM (kg) & $33.30 \pm 6.20$ & $33.07 \pm 5.49$ & 0.752 \\
\hline VFA $\left(\mathrm{cm}^{2}\right)$ & $133.70 \pm 34.46$ & $140.5 \pm 48.59$ & 0.205 \\
\hline $\left.\mathrm{S} / \mathrm{kg} / \mathrm{cm}^{2}\right)$ & $0.26 \pm 0.08$ & $0.27 \pm 0.23$ & 0.760 \\
\hline$W C(\mathrm{~cm})$ & $103.59 \pm 11.78$ & $103.70 \pm 12.74$ & 0.940 \\
\hline $\mathrm{HC}(\mathrm{cm})$ & $110.61 \pm 7.58$ & $110.34 \pm 11.86$ & 0.832 \\
\hline WHR & $0.94 \pm 0.09$ & $0.99 \pm 0.69$ & 0.365 \\
\hline $\mathrm{SBP}(\mathrm{mmHg})$ & $130.88 \pm 12.79$ & $131.73 \pm 13.64$ & 0.607 \\
\hline $\mathrm{DBP}(\mathrm{mmHg})$ & $82.66 \pm 10.22$ & $83.75 \pm 10.70$ & 0.409 \\
\hline $\mathrm{ALT}(\mathrm{U} / \mathrm{L})$ & $44.79 \pm 37.24$ & $38.36 \pm 26.57$ & 0.113 \\
\hline $\mathrm{AST}(\mathrm{U} / \mathrm{L})$ & $27.98 \pm 14.08$ & $26.02 \pm 11.46$ & 0.224 \\
\hline $\mathrm{TC}(\mathrm{mmol} / \mathrm{L})$ & $5.15 \pm 1.05$ & $5.09 \pm 0.84$ & 0.574 \\
\hline $\mathrm{TG}(\mathrm{mmol} / \mathrm{L})$ & $2.16 \pm 1.70$ & $1.98 \pm 1.12$ & 0.307 \\
\hline $\mathrm{HDL}-\mathrm{C}(\mathrm{mmol} / \mathrm{L})$ & $1.11 \pm 0.27$ & $1.22 \pm 1.16$ & 0.296 \\
\hline LDL-C (mmol/L) & $3.19 \pm 0.74$ & $3.17 \pm 0.71$ & 0.839 \\
\hline Lp (a) (mg/L) & $158.20 \pm 172.72$ & $140.05 \pm 145.57$ & 0.365 \\
\hline FPG (mmol/L) & $5.16 \pm 0.75$ & $5.13 \pm 1.08$ & 0.763 \\
\hline $\mathrm{Cr}(\mu \mathrm{mol} / \mathrm{L})$ & $70.25 \pm 15.70$ & $67.57 \pm 15.71$ & 0.176 \\
\hline UA ( $\mu \mathrm{mol} / \mathrm{L})$ & $404.12 \pm 111.32$ & $389.11 \pm 116.72$ & 0.296 \\
\hline FINS (pmol/L) & $157.82 \pm 198.46$ & $135.32 \pm 80.83$ & 0.233 \\
\hline FCP (pmol/L) & $1292.20 \pm 1201.69$ & $1062.05 \pm 454.12$ & $0.048^{*}$ \\
\hline HbA1c (\%) & $5.51 \pm 0.42$ & $5.48 \pm 0.37$ & 0.516 \\
\hline IFG Number (\%) & $11(8.9 \%)$ & $10(7.6 \%)$ & 0.705 \\
\hline $\operatorname{PLT}\left(\times 10^{9}\right)$ & $267.80 \pm 56.57$ & $261.10 \pm 60.25$ & 0.363 \\
\hline $\operatorname{ALB}(g / L)$ & $45.90 \pm 4.26$ & $45.48 \pm 3.96$ & 0.418 \\
\hline NFS & $-3.12 \pm 1.01$ & $-2.91 \pm 1.07$ & 0.111 \\
\hline Fibrosis Severity Scale & & & 0.126 \\
\hline F0-F2 & $119(96.7 \%)$ & $121(92.4 \%)$ & \\
\hline Indeterminant score & $4(3.3 \%)$ & $10(7.6 \%)$ & \\
\hline $\mathrm{F} 3-\mathrm{F} 4$ & 0 & 0 & \\
\hline HOMA-IR & $5.16 \pm 6.15$ & $4.42 \pm 2.78$ & 0.209 \\
\hline
\end{tabular}

$B M I$ Body mass index, $B W$ body weight, $B F P$ body fat percentage, $B F M$ body fat mass, FFM fat-free mass, SMM skeletal muscle mass, VFA visceral fat area, $S / V$ skeletal muscle mass-to-visceral fat area radio, WC waist circumference, $H C$ hip circumference, WHR waist-to-hip ratio, ALT alanine aminotransferase, AST aspartate aminotransferase, TC total cholesterol, TG triglyceride, $H D L-C$ highdensity lipoprotein cholesterol, $L D L-C$ low-density lipoprotein cholesterol, $[L p(a)]$ Lipoprotein(a), Cr creatinine, UA uric acid, FPG fasting plasma glucose, FINS fasting insulin, FCP fasting C peptide, $P L T$ platelet, $A L B$ albumin, $H b A 1 c$ glycated hemoglobin, NFS non-alcoholic fatty liver disease score, IFG impaired fasting glucose, SBP systolic blood pressure, DBP diastolic blood pressure, HOMA-IR homeostasis model assessment of insulin resistance

a Data are represented as mean \pm SD for continuous variables and as percentages for categorical variables

b Independent samples $t$ test and chi-square test were used for continuous and categorical variables, respectively

${ }^{*} p<0.05$ correlated with HDL-C and negatively correlated with LDL-C, SBP, and DBP.

\section{NFS values in tertile subgroups and relationships between baseline NFS and anthropometrical parameters}

Baseline NFS was positively associated with BFM and VFA and negatively correlated with $\mathrm{S} / \mathrm{V}(p<0.05$; Fig. 5 c, $d, e)$. No difference in NFS was observed at 0,3 , and 6 months in both groups. To further validate the changes in NFS, 123 participants in CIMWM group and 131 participants in TMWM group were respectively stratified into three groups based on the tertiles $(-3.41$ and $-2.53)$ of NFS at baseline. NFS of the highest tertile group gradually decreased over the subsequent 6 months to a significantly different extent from baseline $(p<0.05)$. However, in the lowest tertile, a significant increase was observed at 6 months, which only happened in several participants, while, whose values still remained below the high cut-off point (0.676) of liver fibrosis (Fig. 5a, b).

\section{Outcome differences between CIMWM and TMWM groups during the 6-month follow-up}

From 2 months, the difference started to manifest significance between individuals achieving clinically-significant weight loss (defined as weight loss $\geq 5 \%$ ) from two groups $(p=0.003$, Table 2$)$. At 3 months, there were more obvious differences in achievement of clinically-significant weight loss between groups $(p<0.001)$. At 6 months, the significant difference between the CIMWM $(66.7 \%)$ and TMWM (54.2\%) groups was still maintained $(p=0.042)$.

On the other hand, significant changes of BMI, BFP, $\mathrm{BFM}, \mathrm{S} / \mathrm{V}$ and TC from baseline to 6 months were observed between two groups $(p<0.05)$. Our data showed markedly lower BMI, BFM, and TC levels of the CIMWM group compared to the TMWM group during the whole study in spite of all participants transferring to self-monitoring assessment in the final 3 months $(p<0.05)$. S/V was significantly increased from baseline to 3 months in CIMWM group, and maintained during the following 3 months. Increment of S/V in the CIMWM group was significantly better than that in the TMWM group from 2 to 6 months $(p<0.05)$.

Subjects in the CIMWM group lost more body weight than those in the TMWM group at 3 months $(-8.09 \mathrm{~kg}$ vs. $-5.77 \mathrm{~kg} ; p=0.004)$, but this trend disappeared at 6 months $(-7.42 \mathrm{~kg}$ vs. $-6.86 \mathrm{~kg} ; p=0.461)$. However, there was still a marked difference in achievement of clinically significant weight loss between the CIMWM and TMWM groups at 6 months $(p=0.042)$. BMI in the CIMWM group decreased significantly by $2.08 \mathrm{~kg} / \mathrm{m}^{2}$ at 2 months, $2.58 \mathrm{~kg} / \mathrm{m}^{2}$ at 3 months, and $2.44 \mathrm{~kg} / \mathrm{m}^{2}$ at 6 months, compared to the TMWM group (2 months: 

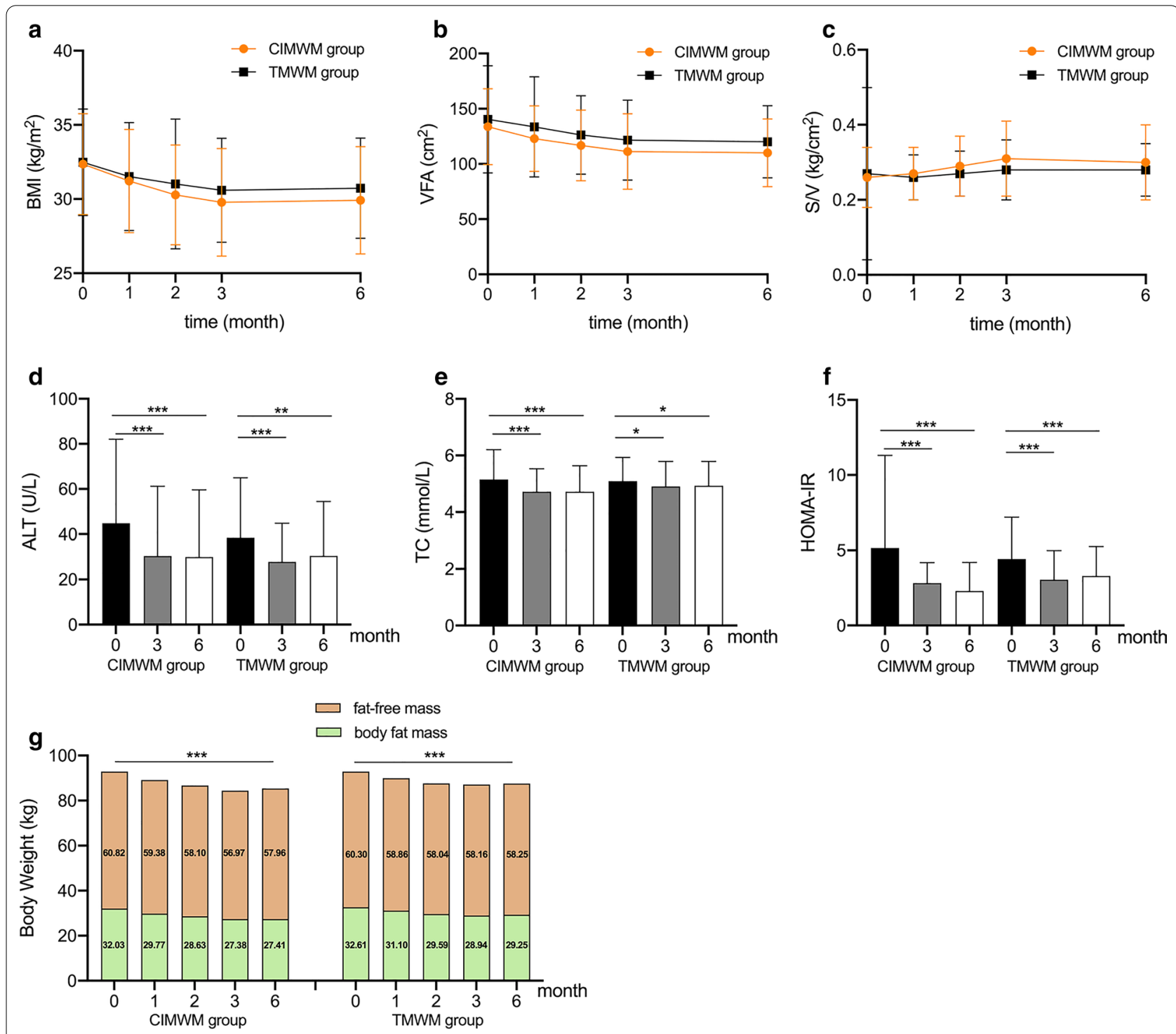

Fig. $3 \mathrm{BMI}, \mathrm{VFA}, \mathrm{S} / \mathrm{N}, \mathrm{ALT}, \mathrm{TC}, \mathrm{HOMA}$-IR, BFM and FFM changes in CIMWM and TMWM groups. ${ }^{*} p<0.05,{ }^{* *} p<0.01,{ }^{* * *} p<0.001$, body mass index (BMI), visceral fat area (VFA), skeletal muscle mass-to-visceral fat area radio (SN), alanine aminotransferase (ALT), total cholesterol (TC), homeostasis model assessment of insulin resistance (HOMA-IR), body fat mass (BFM), fat-free mass (FFM), Companion-Intensive Multi-aspect Weight Management (CIMWM), Traditional Multi-aspect Weight Management (TMWM). a Changes in BMl from baseline to 6 months respectively in CIMWM and TMWM. $\mathbf{b}$ Changes in VFA from baseline to 6 months respectively in CIMWM and TMWM. $\mathbf{c}$ Changes in S/N from baseline to 6 months respectively in CIMWM and TMWM. $\mathbf{d}$ Changes in ALT from baseline to 6 months respectively in CIMWM and TMWM. e Changes in TC from baseline to 6 months respectively in CIMWM and TMWM. $\mathbf{f}$ Changes in HOMA-IR from baseline to 6 months respectively in CIMWM and TMWM. g BFM and FFM reduction from baseline to 6 months respectively in CIMWM and TMWM

$1.46 \mathrm{~kg} / \mathrm{m}^{2}, 3$ months: $1.88 \mathrm{~kg} / \mathrm{m}^{2}, 6$ months, $1.74 \mathrm{~kg} /$ $\mathrm{m}^{2} ; p<0.05$, Table 2). We also found greater reduction of VFA in the CIMWM group at 3 months compared to the TMWM group, which was not maintained until the end of our research.

\section{Discussion}

Results from this 6-month, randomized, controlled trial on Chinese adults with obesity showed that weight management with regular guidance by physicians, dietitians, and health managers is feasible and effective for improvement of body weight, BMI, body composition parameters (BFM and VFA), liver function, blood lipid 


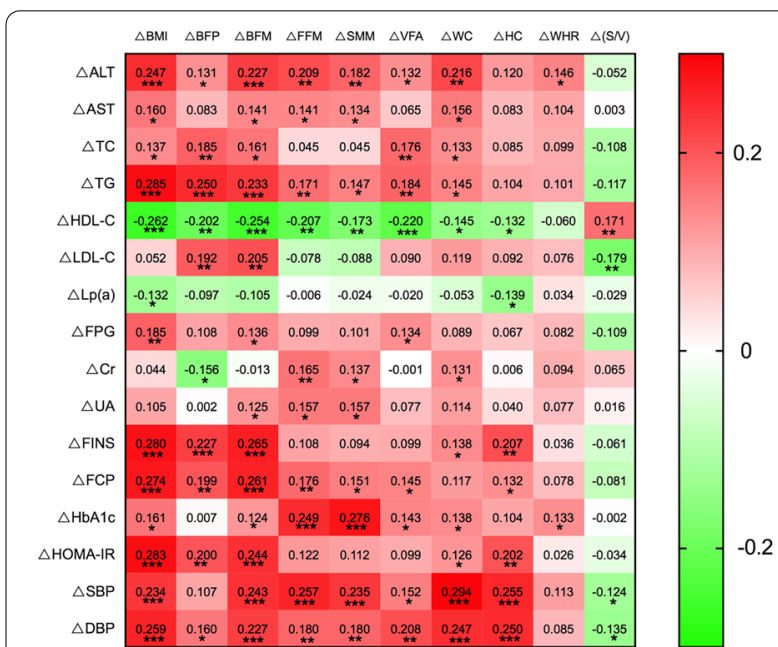

Fig. 4 Spearman's correlation coefficient heatmap among physical and hematological indices changes from baseline to 6 months. Body mass index (BMI), body fat percentage (BFP), body fat mass (BFM), fat-free mass (FFM), skeletal muscle mass (SMM), visceral fat area (VFA), skeletal muscle mass-to-visceral fat area radio (SN), waist circumference (WC), hip circumference (HC), waist-to-hip ratio (WHR), alanine aminotransferase (ALT), aspartate aminotransferase (AST), total cholesterol (TC), triglyceride (TG), high-density lipoprotein cholesterol (HDL-C), low-density lipoprotein cholesterol (LDL-C), lipoprotein(a) [Lp(a)], creatinine (Cr), uric acid (UA), fasting plasma glucose (FPG), fasting insulin (FINS), fasting C peptide (FCP), glycated hemoglobin (HbA1c), systolic blood pressure (SBP), diastolic blood pressure (DBP), homeostasis model assessment of insulin resistance (HOMA-IR). ${ }^{*} p<0.05,{ }^{* *} p<0.01 ;{ }^{* * *} p<0.001$

profiles, and insulin resistance in all participants. The CIMWM strategy involving both daily online instructions and monthly face-to-face guidance was superior to TMWM with respect to improvement of BMI, TC, and body composition parameters, such as BFM, VFA, and $\mathrm{S} / \mathrm{V}$. Individuals subjected to the CIMWM program showed better adherence to guidelines and reprogramming of lifestyle. Efforts to estimate body composition changes during interventions are important in improving our awareness of metabolic health. Metabolic memory in terms of continuous reduction of BMI, BFM, and TC was retained up to 6 months, even with transfer of participants in the CIMWM group to the self-monitoring phase in the last 3 months. NFS was further evaluated to determine the degree of liver fibrosis. Our data suggest that weight management program is more beneficial in decreasing NFS among subjects with basal NFS levels in the highest tertile.

The primary outcomes of this study were the clinically-significant weight loss and reduction of BMI, BFM, and VFA from baseline to 6-month follow-up. The CIMWM group achieved significantly better reduction of these parameters than the TMWM group throughout the study. Notably, body weight increased slightly at 6 months, compared to 3 months, in both groups, but remained lower than that at baseline. We believe that the weight gain in the CIMWM group during the last 3 months of the study is attributable to the transfer to self-monitoring. Another potential reason suggested by Burke et al. [18] is a gradual decline in adherence to self-monitoring weight management program, which is common during the follow-up period. But even in the last 3 months of self-monitoring period, CIMWM group still achieved significantly better weight loss than TMWM group, suggesting the previous intense online intervention paved the way for a sustainable and positive dietary, exercise and lifestyle in their subsequent lives.

The change in body composition after weight management is an interesting phenomenon. Total body fat and distribution, especially visceral fat, is known to be associated with higher risk of metabolic syndrome [19]. Here, our data showed a reduction of BFP, BFM, and VFA in all participants, along with FFM and SMM showing a mild decrease despite physical activity support. FFM reduction is clearly associated with poor metabolic state [20-22]. Meanwhile, VFA increase and SMM decrease appear closely related to aggravation of insulin resistance and progression of NAFLD [23]. Therefore, another recommended index is the SMM to VFA radio $(S / V)$, which simultaneously describes variations in both parameters [23]. A significant increase in $S / V$ in all subjects was observed during the whole intervention process. Moreover, from 2 to 6 months, the increase of S/V in CIMWM group was significantly higher than that in TMWM group, suggesting the better effects of CIMWM than TMWM in the improvement of glucose metabolism and liver fat metabolism.

NFS is a clinical indicator of liver fibrosis [24]. Individuals with basal NFS in the highest tertile displayed significant reduction in NFS vaules after 6 months of weight intervention, indicating that our medical weight management strategy is effective not only for weight loss but also for the improvement of NAFLD. NFS in subjects starting in the lowest tertile was slightly increased, but remained under the 0.676 cut-off point for liver fibrosis. This finding is possibly related to the rebound of BMI among those individuals. However, plasma ALT and AST concentrations are still regarded as better clinical indicators than other noninvasive biomarkers/scores for definitive diagnosis of nonalcoholic steatohepatitis (NASH) and/ or advanced fibrosis in patients with T2DM [25]. And we observed a significant reduction in ALT and AST levels in all participants after the intervention, which together with the NFS, ensured a well enough liver condition in this study. 


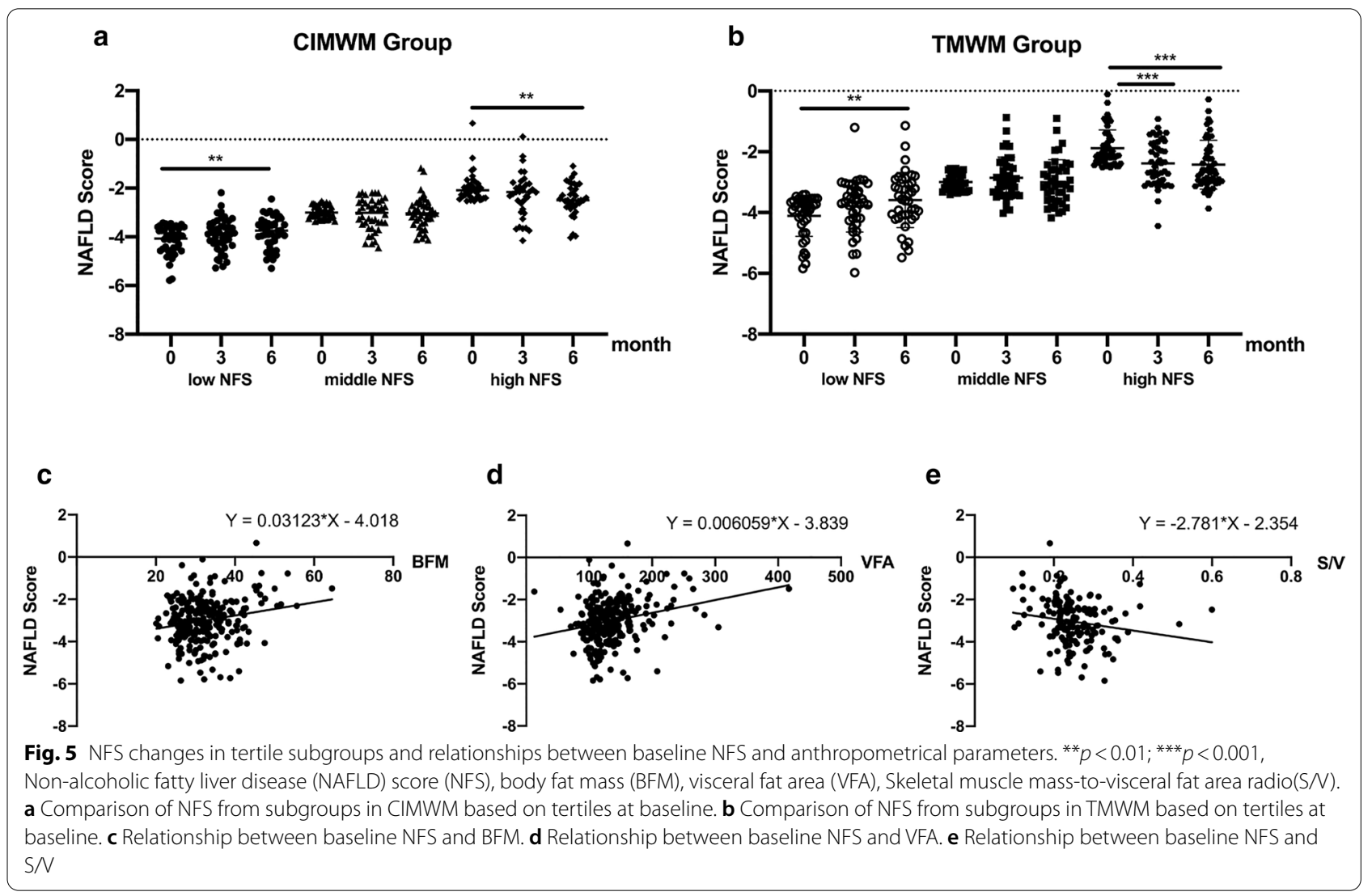

At baseline, a negative correlation was observed between NFS and S/V. S/V is reported to be an independent factor that affects the controlled attenuation parameter (CAP) reflecting hepatic fat accumulation and liver stiffness measurements (LSM), representing hepatic inflammation and fibrosis [23]. In another study on NAFLD patients [24], low S/V indicated poorer hepatic condition, confirmed with liver transient elastography. Accumulating basic and clinical studies have focused on cross-talk among muscle, fat, and liver components [26]. A recent review suggests that adipose tissue function is a critical driver of NAFLD and NASH. Cytokines and hormones secreted by adipose tissue could affect the liver by regulating the lipid flux and affecting hepatocyte function via exosomal signaling [27]. Skeletal muscle is also regarded as an endocrine organ [28] that regulates myokine secretion. Circulating myonectin linking skeletal muscle to lipid metabolism in the liver and adipose tissue provides insights into tissue cross-talk, which underlies the integrated control of whole-body metabolism [29]. Hepatoprotective IL-6 [30] and irisin [31] are further reported to improve hepatic steatosis [32]. Above all, cross-talk among muscle, fat and liver might be an interesting direction for our future research on obesity.
Our study has some limitations that should be taken into consideration. Evaluation of liver steatosis and fibrosis with FibroTouch, FibroScan, or magnetic resonance elastography may present more efficient and precise results, which we didn't get a chance to obtain. In addition, we were not able to develop an accurate method to collect data on frequency and intensity of physical activities from our subjects, which may give more information on the exercise aspect for intensive lifestyle modification.

\section{Conclusion}

Our study on weight management in Chinese adults with obesity showed that the CIMWM approach is more effective than the TMWM approach, leading to greater improvement of clinically-significant weight loss, BMI, BFM, VFA, S/V and TC parameters. The CIMWM strategy was particularly effective in motivating patient adherence to the intervention and lifestyle reprogramming. Metabolic memory in terms of continuous reduction of BMI, BFM, and TC was retained up to 6 months despite participants being transferred to the self-monitoring phase during the last 3 months of the study. Our results support the potential of a face-to-face program coupled with an online intervention tool in achieving scientifically 
Table 2 Outcome differences between CIMWM and TMWM groups during the 6-month follow-up period ${ }^{\mathrm{a}}$

\begin{tabular}{|c|c|c|c|c|}
\hline & $\begin{array}{l}\text { Time } \\
\text { (month) }\end{array}$ & CIMWM & TMWM & $p$ value $^{b}$ \\
\hline \multirow[t]{4}{*}{ Weight loss $\geq 5 \%$} & 1 & $30.9 \%(38 / 123)$ & $24.4 \%(32 / 131)$ & 0.249 \\
\hline & 2 & $68.3 \%(84 / 123)$ & $49.6 \%(65 / 131)$ & $0.003^{* *}$ \\
\hline & 3 & $76.4 \%(94 / 123)$ & $54.2 \%(71 / 131)$ & $<0.001^{* * *}$ \\
\hline & 6 & $66.7 \%(82 / 123)$ & $54.2 \%(71 / 131)$ & $0.042^{*}$ \\
\hline \multirow[t]{4}{*}{$\triangle B M I\left(k g / m^{2}\right)$} & 1 & $-1.14 \pm 1.17$ & $-0.96 \pm 1.05$ & 0.437 \\
\hline & 2 & $-2.08 \pm 1.21$ & $-1.46 \pm 3.84$ & $0.013^{*}$ \\
\hline & 3 & $-2.58 \pm 1.51$ & $-1.88 \pm 2.12$ & $0.006^{* *}$ \\
\hline & 6 & $-2.44 \pm 1.82$ & $-1.74 \pm 2.59$ & $0.006^{* *}$ \\
\hline \multirow[t]{4}{*}{$\triangle B W(k g)$} & 1 & $-3.65 \pm 2.31$ & $-3.06 \pm 3.70$ & 0.438 \\
\hline & 2 & $-6.30 \pm 5.62$ & $-5.42 \pm 7.55$ & 0.260 \\
\hline & 3 & $-8.09 \pm 6.57$ & $-5.77 \pm 7.90$ & $0.004^{* *}$ \\
\hline & 6 & $-7.42 \pm 8.16$ & $-6.86 \pm 10.67$ & 0.461 \\
\hline \multirow[t]{4}{*}{$\triangle B F P(\%)$} & 1 & $-1.13 \pm 1.50$ & $-0.60 \pm 1.45$ & 0.062 \\
\hline & 2 & $-1.82 \pm 2.14$ & $-1.33 \pm 2.46$ & 0.085 \\
\hline & 3 & $-2.43 \pm 2.77$ & $-1.89 \pm 2.52$ & 0.058 \\
\hline & 6 & $-2.74 \pm 3.26$ & $-1.94 \pm 3.02$ & $0.006^{* *}$ \\
\hline \multirow[t]{4}{*}{$\triangle B F M(k g)$} & 1 & $-2.26 \pm 1.79$ & $-1.51 \pm 1.72$ & 0.055 \\
\hline & 2 & $-3.40 \pm 3.13$ & $-3.02 \pm 4.20$ & 0.284 \\
\hline & 3 & $-4.64 \pm 2.80$ & $-3.67 \pm 4.21$ & $0.015^{*}$ \\
\hline & 6 & $-4.62 \pm 3.54$ & $-3.36 \pm 5.67$ & $0.002^{* *}$ \\
\hline \multirow[t]{4}{*}{$\triangle F F M(k g)$} & 1 & $-1.44 \pm 1.86$ & $-1.43 \pm 3.15$ & 0.873 \\
\hline & 2 & $-2.73 \pm 4.73$ & $-2.25 \pm 4.99$ & 0.498 \\
\hline & 3 & $-3.86 \pm 6.52$ & $-2.14 \pm 5.22$ & $0.004^{* *}$ \\
\hline & 6 & $-2.86 \pm 5.59$ & $-2.05 \pm 5.15$ & 0.203 \\
\hline \multirow[t]{4}{*}{$\triangle S M M(k g)$} & 1 & $-0.78 \pm 1.41$ & $-0.80 \pm 1.72$ & 0.871 \\
\hline & 2 & $-1.29 \pm 2.40$ & $-1.21 \pm 2.68$ & 0.925 \\
\hline & 3 & $-1.08 \pm 4.47$ & $-1.11 \pm 2.60$ & 0.855 \\
\hline & 6 & $-2.06 \pm 4.71$ & $-1.48 \pm 3.63$ & 0.149 \\
\hline \multirow[t]{4}{*}{$\triangle \mathrm{VFA}\left(\mathrm{cm}^{2}\right)$} & 1 & $-10.86 \pm 19.05$ & $-6.84 \pm 25.88$ & $0.028^{*}$ \\
\hline & 2 & $-17.00 \pm 21.87$ & $-14.13 \pm 36.58$ & 0.067 \\
\hline & 3 & $-22.38 \pm 23.52$ & $-18.90 \pm 36.28$ & $0.043^{*}$ \\
\hline & 6 & $-23.70 \pm 28.21$ & $-20.45 \pm 39.15$ & 0.051 \\
\hline \multirow[t]{4}{*}{$\triangle(S / N)\left(\mathrm{kg} / \mathrm{cm}^{2}\right)$} & 1 & $0.01 \pm 0.05$ & $-0.01 \pm 0.22$ & 0.061 \\
\hline & 2 & $0.03 \pm 0.06$ & $-0.00 \pm 0.22$ & $0.012^{*}$ \\
\hline & 3 & $0.05 \pm 0.07$ & $0.01 \pm 0.23$ & $0.002^{* *}$ \\
\hline & 6 & $0.04 \pm 0.08$ & $0.01 \pm 0.22$ & $0.008^{* *}$ \\
\hline \multirow[t]{4}{*}{$\triangle W C(\mathrm{~cm})$} & 1 & $-2.29 \pm 7.22$ & $-1.35 \pm 9.95$ & 0.322 \\
\hline & 2 & $-6.14 \pm 11.56$ & $-4.19 \pm 10.39$ & $0.047^{*}$ \\
\hline & 3 & $-7.12 \pm 7.80$ & $-5.35 \pm 11.78$ & 0.071 \\
\hline & 6 & $-7.33 \pm 8.93$ & $-5.70 \pm 12.25$ & 0.095 \\
\hline \multirow[t]{4}{*}{$\triangle H C(\mathrm{~cm})$} & 1 & $-2.13 \pm 4.46$ & $-0.76 \pm 9.84$ & 0.218 \\
\hline & 2 & $-4.34 \pm 5.45$ & $-4.40 \pm 14.24$ & 0.833 \\
\hline & 3 & $-5.71 \pm 5.81$ & $-4.39 \pm 13.94$ & 0.241 \\
\hline & 6 & $-4.95 \pm 5.48$ & $-3.83 \pm 11.09$ & 0.329 \\
\hline \multirow[t]{4}{*}{$\triangle W H R$} & 1 & $-0.00 \pm 0.07$ & $-0.06 \pm 0.69$ & 0.931 \\
\hline & 2 & $-0.02 \pm 0.10$ & $-0.04 \pm 0.72$ & 0.936 \\
\hline & 3 & $-0.02 \pm 0.08$ & $-0.05 \pm 0.72$ & 0.164 \\
\hline & 6 & $-0.03 \pm 0.08$ & $-0.07 \pm 0.70$ & 0.936 \\
\hline \multirow[t]{3}{*}{$\triangle \mathrm{SBP}(\mathrm{mmHg})$} & 1 & $-4.92 \pm 11.27$ & $-4.49 \pm 12.44$ & 0.483 \\
\hline & 2 & $-6.38 \pm 12.33$ & $-4.91 \pm 13.47$ & 0.136 \\
\hline & 3 & $-7.11 \pm 13.17$ & $-7.21 \pm 12.97$ & 0.772 \\
\hline
\end{tabular}

Table 2 (continued)

\begin{tabular}{|c|c|c|c|c|}
\hline & $\begin{array}{l}\text { Time } \\
\text { (month) }\end{array}$ & CIMWM & TMWM & $p$ value $^{b}$ \\
\hline & 6 & $-6.66 \pm 12.97$ & $-6.32 \pm 13.64$ & 0.531 \\
\hline \multirow[t]{4}{*}{$\triangle \mathrm{DBP}(\mathrm{mmHg})$} & 1 & $-4.62 \pm 10.17$ & $-4.33 \pm 10.31$ & 0.305 \\
\hline & 2 & $-5.94 \pm 9.98$ & $-4.34 \pm 9.83$ & $0.017^{*}$ \\
\hline & 3 & $-6.24 \pm 10.19$ & $-5.73 \pm 10.53$ & 0.208 \\
\hline & 6 & $-5.64 \pm 10.25$ & $-6.14 \pm 10.52$ & 0.829 \\
\hline \multirow[t]{2}{*}{$\triangle \mathrm{ALT}(\mathrm{U} / \mathrm{L})$} & 3 & $-14.40 \pm 21.74$ & $-10.61 \pm 24.03$ & 0.836 \\
\hline & 6 & $-14.86 \pm 25.98$ & $-7.94 \pm 31.41$ & 0.156 \\
\hline \multirow[t]{2}{*}{$\triangle \mathrm{AST}(\mathrm{U} / \mathrm{L})$} & 3 & $-6.32 \pm 10.49$ & $-4.50 \pm 10.08$ & 0.548 \\
\hline & 6 & $-5.62 \pm 12.86$ & $-2.48 \pm 14.37$ & 0.095 \\
\hline \multirow[t]{2}{*}{$\triangle \mathrm{TC}(\mathrm{mmol} / \mathrm{L})$} & 3 & $-0.43 \pm 0.94$ & $-0.18 \pm 0.85$ & $0.025^{*}$ \\
\hline & 6 & $-0.43 \pm 0.97$ & $-0.16 \pm 0.85$ & $0.013^{*}$ \\
\hline \multirow[t]{2}{*}{$\triangle \mathrm{TG}(\mathrm{mmol} / \mathrm{L})$} & 3 & $-0.76 \pm 1.79$ & $-0.60 \pm 0.96$ & 0.849 \\
\hline & 6 & $-0.48 \pm 1.63$ & $-0.30 \pm 1.17$ & 0.713 \\
\hline \multirow[t]{2}{*}{$\triangle H D L-C(\mathrm{mmol} / \mathrm{L})$} & 3 & $0.03 \pm 0.23$ & $0.02 \pm 1.42$ & 0.339 \\
\hline & 6 & $0.10 \pm 0.27$ & $0.02 \pm 1.15$ & 0.283 \\
\hline \multirow[t]{2}{*}{$\triangle \mathrm{LDL}-\mathrm{C}(\mathrm{mmol} / \mathrm{L})$} & 3 & $-0.17 \pm 0.65$ & $-0.06 \pm 0.72$ & 0.168 \\
\hline & 6 & $-0.27 \pm 0.67$ & $-0.15 \pm 0.72$ & 0.126 \\
\hline \multirow[t]{2}{*}{$\triangle \mathrm{Lp}(\mathrm{a})(\mathrm{mg} / \mathrm{L})$} & 3 & $42.88 \pm 149.61$ & $49.15 \pm 86.74$ & 0.881 \\
\hline & 6 & $47.91 \pm 193.69$ & $25.69 \pm 94.77$ & 0.122 \\
\hline \multirow[t]{2}{*}{$\triangle F P G(m m o l / L)$} & 3 & $-0.26 \pm 0.74$ & $-0.19 \pm 1.06$ & 0.674 \\
\hline & 6 & $-0.23 \pm 0.91$ & $-0.18 \pm 1.14$ & 0.756 \\
\hline \multirow[t]{2}{*}{$\triangle \mathrm{Cr}(\mu \mathrm{mol} / \mathrm{L})$} & 3 & $-1.30 \pm 9.39$ & $0.70 \pm 11.46$ & 0.448 \\
\hline & 6 & $-0.61 \pm 11.08$ & $-0.97 \pm 14.54$ & 0.282 \\
\hline \multirow[t]{2}{*}{$\triangle \cup A(\mu \mathrm{mol} / \mathrm{L})$} & 3 & $5.25 \pm 71.00$ & $-3.48 \pm 102.55$ & 0.097 \\
\hline & 6 & $-34.21 \pm 82.14$ & $-19.11 \pm 95.79$ & 0.361 \\
\hline \multirow[t]{2}{*}{$\triangle F I N S(p m o l / L)$} & 3 & $-67.95 \pm 192.05$ & $-39.86 \pm 76.70$ & 0.229 \\
\hline & 6 & $-62.95 \pm 194.36$ & $-32.24 \pm 73.93$ & 0.107 \\
\hline \multirow[t]{2}{*}{$\triangle F C P(p m o l / L)$} & 3 & $-385.4 \pm 1244.23$ & $-120.41 \pm 698.46$ & 0.382 \\
\hline & 6 & $-421.05 \pm 1219.72$ & $-197.40 \pm 450.50$ & 0.872 \\
\hline \multirow[t]{2}{*}{$\triangle \mathrm{HbA1c}(\%)$} & 3 & $-0.17 \pm 0.31$ & $-0.09 \pm 0.36$ & 0.117 \\
\hline & 6 & $-0.04 \pm 0.41$ & $-0.02 \pm 0.45$ & 0.885 \\
\hline \multirow[t]{2}{*}{$\triangle \mathrm{HOMA-IR}$} & 3 & $-2.35 \pm 6.01$ & $-1.37 \pm 2.76$ & 0.182 \\
\hline & 6 & $-2.17 \pm 6.08$ & $-1.12 \pm 2.74$ & 0.103 \\
\hline \multirow[t]{2}{*}{$\triangle N F S$} & 3 & $0.01 \pm 0.71$ & $-0.10 \pm 0.86$ & 0.749 \\
\hline & 6 & $-0.02 \pm 0.74$ & $-0.03 \pm 1.02$ & 0.649 \\
\hline
\end{tabular}

$B M I$ body mass index, $B W$ body weight, $B F P$ body fat percentage, $B F M$ body fat mass, FFM fat-free mass, SMM skeletal muscle mass, VFA visceral fat area, $S N$ skeletal muscle mass-to-visceral fat area radio, WC waist circumference, $H C$ hip circumference, WHR waist-to-hip ratio, ALT alanine aminotransferase, AST aspartate aminotransferase, $T C$ total cholesterol, $T G$ triglyceride, $H D L-C$ highdensity lipoprotein cholesterol, $L D L-C$ low-density lipoprotein cholesterol, $[L p(a)]$ Lipoprotein(a), Cr creatinine, UA uric acid, FPG fasting plasma glucose, FINS fasting insulin, $F C P$ fasting $C$ peptide, $H b A 1 c$ glycated hemoglobin, NFS non-alcoholic fatty liver disease score, $S B P$ systolic blood pressure, $D B P$ diastolic blood pressure HOMA-IR homeostasis model assessment of insulin resistance

a Data are represented as mean \pm SD for continuous variables and as percentages for categorical variables

b Between-group differences were analyzed using mixed model controlling for baseline index; categorical variables of individuals with weight loss $\geq 5 \%$ were analyzed using chi-square analysis

${ }^{*} p<0.05,{ }^{* *} p<0.01,{ }^{* * *} p<0.001$ 
effective management of metabolic syndrome, and providing a positive contribution to national health.

\section{Abbreviations}

BMI: Body mass index; BW: Body weight; BFP: Body fat percentage; BFM: Body fat mass; FFM: Fat-free mass; SMM: Skeletal muscle mass; VFA: Visceral fat area; SN: Skeletal muscle mass-to-visceral fat area radio; WC: Waist circumference; HC: Hip circumference; WHR: Waist-to-hip ratio; SBP: Systolic blood pressure; DBP: Diastolic blood pressure; FPG: Fasting plasma glucose; ALT: Alanine aminotransferase; AST: Aspartate aminotransferase; TC: Total cholesterol; TG: Triglyceride; HDL-C: High-density lipoprotein cholesterol; LDL-C: Low-density lipoprotein cholesterol; Lp(a): Lipoprotein(a); Cr: Creatinine; UA: Uric acid: PLT: Platelet; ALB: Albumin; FINS: Fasting insulin; FCP: Fasting C peptide; HbA1c: Glycated hemoglobin; NFS: Non-alcoholic fatty liver disease score; HOMA-IR: Homeostasis model assessment of insulin resistance; IFG: Impaired fasting glucose.
\end{abstract}

\section{Supplementary information}

Supplementary information accompanies this paper at https://doi. org/10.1186/s12986-020-00511-6.

Additional file 1: Table S1. Anthropometric, metabolic, and clinical characteristics of participants in each group before and after the weight loss intervention.

\section{Acknowledgements}

The following contributors assisted with patient recruitment, intervention and/or data collection during the trial: The First Affiliated Hospital of Nanjing Medical University (Baowen Yu, Chenyan Lu), The Affiliated Changzhou No.2 People's Hospital of Nanjing Medical University (Ping Yang, Xiaoya Gao), The First Affiliated Hospital of Soochow University (Shengyi Zou), Suzhou Municipal Hospital Affiliated to Nanjing Medical University (Ying Wu), and Affiliated Hospital of Jiangsu University (Guoyue Yuan). We are grateful for their assistance with advice on ethical issues, good clinical practice, and approval of the study protocol. Additionally, we thank all other colleagues involved in this research for their valuable input, as well as all the participants in this study.

\section{Authors' contributions}

WJ conducted data collection as well as analysis and took the lead in writing the manuscript. SH conceived and designed the study, conducted data collection, and contributed to the final version of the manuscript. SM conducted data collection, helped with data analysis and revision of the manuscript. JB contributed to statistical analysis. LC, BS and XY made contributions on recruitment of participants and data collection. $\mathrm{HZ}$ designed and conducted the study, contributed to data interpretation and manuscript revision, and provided medical supervision. All co-authors contributed to implementation of the clinical trial and design of the protocol for intervention. $\mathrm{HZ}$ is the guarantor of this work and, as such, has full access to all the data and takes responsibility for data integrity and accuracy of analysis. All authors read and approved the final manuscript.

\section{Funding}

This study was supported by the National Key R\&D Program of China [2018YFA0506904], the Major Research plan of the National Natural Science Foundation of China [91854122], the National Natural Science Foundation of China [81670723], and Revitalize and Defend The Key Talents Subsidy Project from the Science and Education Department of Public Health of Jiangsu Province [ZDRCA2016017] awarded to Dr. Hongwen Zhou.

\section{Availability of data and materials}

The datasets used and/or analyzed during the current study are available from the corresponding author on reasonable request.

\section{Ethics approval and consent to participate}

This study was approved by the Ethics Committee at the First Affiliated Hospital of Nanjing Medical University (Nanjing, Jiangsu, China) (2018-SR-069). Written informed consents were obtained from all participants.

\section{Consent for publication}

Not applicable.

\section{Competing interests}

Our research has received funding from Guangzhou ND-fit Nutrition and Health Consulting Co. Ltd, with no personal remuneration. The company has no interests in this program. The authors declare no competing interests.

\section{Author details}

${ }^{1}$ Department of Endocrinology and Metabolism, The First Affiliated Hospital of Nanjing Medical University, Jiangsu Province Hospital, Nanjing 210029, China. ${ }^{2}$ Department of Geriatrics, The Affiliated Hospital of Nantong University, Nantong 226001, China. ${ }^{3}$ Department of Endocrinology and Metabolism, The Affiliated Huai'an No.1 People's Hospital of Nanjing Medical University, Huai'an 223001, China. ${ }^{4}$ Department of Endocrinology, The Second People's Hospital of Huai'an, The Affiliated Huai'an Hospital of Xuzhou Medical University, Huai'an 223001, China. ${ }^{5}$ Department of Endocrinology, The Affiliated Suzhou Science \& Technology Town Hospital of Nanjing Medical University, Suzhou 215000, China. ${ }^{6}$ Department of Endocrinology, The Affiliated Hospital of Jiangsu University, Zhenjiang 212000, China. ${ }^{7}$ Department of Endocrinology, The First People's Hospital of Chuzhou, Chuzhou 239000, China. ${ }^{8}$ Department of Endocrinology, Northern Jiangsu People's Hospital, Yangzhou University, Yangzhou 225000, China. ${ }^{9}$ Department of Endocrinology, The Second Affiliated Hospital of Dalian Medical University, Dalian 116000, China ${ }^{10}$ Department of Biostatistics, School of Public Health, Nanjing Medical University, Nanjing 210029, China. ${ }^{11}$ Department of Endocrinology, Suzhou Municipal Hospital Affiliated to Nanjing Medical University, Suzhou 215000, China. ${ }^{12}$ Department of Endocrinology, The First Affiliated Hospital of Soochow University, Suzhou 215000, China. ${ }^{13}$ Department of Endocrinology, The Affiliated Changzhou No. 2 People's Hospital of Nanjing Medical University, Changzhou 213000, China.

Received: 30 May 2020 Accepted: 8 October 2020 Published online: 03 February 2021

\section{References}

1. Kelly T, Yang W, Chen CS, Reynolds K, He J. Global burden of obesity in 2005 and projections to 2030. Int J Obes (Lond). 2008;32(9):1431-7.

2. Xi B, Liang $Y$, He T, Reilly $K H, H u Y$, Wang $Q$, et al. Secular trends in the prevalence of general and abdominal obesity among Chinese adults, 1993-2009. Obes Rev. 2012;13(3):287-96.

3. Kopelman PG. Obesity as a medical problem. Nature. 2000;404(6778):635-43.

4. Xu Y, Li H, Wang A, Su Z, Yang G, Luo Y, et al. Association between the metabolically healthy obese phenotype and the risk of myocardial infarction: results from the Kailuan study. Eur J Endocrinol. 2018;179(6):343-52.

5. Visscher TL, Seidell JC. The public health impact of obesity. Annu Rev Public Health. 2001;22:355-75.

6. Singh AK, Singh R. Pharmacotherapy in obesity: a systematic review and meta-analysis of randomized controlled trials of anti-obesity drugs. Expert Rev Clin Pharmacol. 2020;13(1):53-64.

7. Watson S, Woodside JV, Ware LJ, Hunter SJ, McGrath A, Cardwell CR, et al. Effect of a web-based behavior change program on weight loss and cardiovascular risk factors in overweight and obese adults at high risk of developing cardiovascular disease: randomized controlled trial. J Med Internet Res. 2015;17(7):e177.

8. Jimenez V, Jambrina C, Casana E, Sacristan V, Munoz S, Darriba S, et al. FGF21 gene therapy as treatment for obesity and insulin resistance. EMBO Mol Med. 2018;10(8):e8791.

9. Jacobs S, Radnitz C, Hildebrandt T. Adherence as a predictor of weight loss in a commonly used smartphone application. Obes Res Clin Pract. 2017;11(2):206-14.

10. Lean ME, Leslie WS, Barnes AC, Brosnahan N, Thom G, McCombie L, et al. Primary care-led weight management for remission of type 2 
diabetes (DiRECT): an open-label, cluster-randomised trial. Lancet. 2018;391(10120):541-51.

11. van Velthoven MH, Li Y, Wang W, Chen L, Du X, Wu Q, et al. Prevalence of mobile phones and factors influencing usage by caregivers of young children in daily life and for health care in rural China: a mixed methods study. PLoS ONE. 2015;10(3):e0116216.

12. Li Y, Hu X, Zhang Q, Liu A, Fang H, Hao L, et al. The nutrition-based comprehensive intervention study on childhood obesity in China (NISCOC): a randomised cluster controlled trial. BMC Public Health. 2010;10:229.

13. Yoshimura E, Kumahara H, Tobina T, Matsuda T, Ayabe M, Kiyonaga A, et al. Lifestyle intervention involving calorie restriction with or without aerobic exercise training improves liver fat in adults with visceral adiposity. J Obes. 2014;2014:197216.

14. Angulo P, Hui JM, Marchesini G, Bugianesi E, George J, Farrell GC, et al. The NAFLD fibrosis score: a noninvasive system that identifies liver fibrosis in patients with NAFLD. Hepatology. 2007;45(4):846-54.

15. Richter B, Hemmingsen B, Metzendorf MI, Takwoingi Y. Development of type 2 diabetes mellitus in people with intermediate hyperglycaemia. Cochrane Database Syst Rev. 2018;10:12661.

16. Ross KM, Wing RR. Impact of newer self-monitoring technology and brief phone-based intervention on weight loss: a randomized pilot study. Obesity (Silver Spring). 2016;24(8):1653-9.

17. Tate DF, Jackvony EH, Wing RR. A randomized trial comparing human e-mail counseling, computer-automated tailored counseling, and no counseling in an Internet weight loss program. Arch Intern Med. 2006;166(15):1620-5.

18. Burke LE, Wang J, Sevick MA. Self-monitoring in weight loss: a systematic review of the literature. J Am Diet Assoc. 2011;111(1):92-102.

19. Koster A, Stenholm S, Alley DE, Kim LJ, Simonsick EM, Kanaya AM, et al. Body fat distribution and inflammation among obese older adults with and without metabolic syndrome. Obesity (Silver Spring). 2010;18(12):2354-61.

20. Atlantis E, Martin SA, Haren MT, Taylor AW, Wittert GA, Members of the Florey Adelaide Male Ageing S. Inverse associations between muscle mass, strength, and the metabolic syndrome. Metabolism. 2009;58(7):1013-22.

21. Srikanthan P, Karlamangla AS. Relative muscle mass is inversely associated with insulin resistance and prediabetes. Findings from the third National Health and Nutrition Examination Survey. J Clin Endocrinol Metab. 2011;96(9):2898-903.

22. Vink RG, Roumans NJ, Arkenbosch LA, Mariman EC, van Baak MA. The effect of rate of weight loss on long-term weight regain in adults with overweight and obesity. Obesity (Silver Spring). 2016;24(2):321-7.
23. Shida T, Akiyama K, Oh S, Sawai A, Isobe T, Okamoto Y, et al. Skeletal muscle mass to visceral fat area ratio is an important determinant affecting hepatic conditions of non-alcoholic fatty liver disease. J Gastroenterol. 2018;53(4):535-47.

24. Shida T, Oshida N, Oh S, Okada K, Shoda J. Progressive reduction in skeletal muscle mass to visceral fat area ratio is associated with a worsening of the hepatic conditions of non-alcoholic fatty liver disease. Diabetes Metab Syndr Obes. 2019;12:495-503.

25. Bril F, McPhaul MJ, Caulfield MP, Clark VC, Soldevilla-Pico C, Firpi-Morell RJ, et al. Performance of plasma biomarkers and diagnostic panels for nonalcoholic steatohepatitis and advanced fibrosis in patients with type 2 diabetes. Diabetes Care. 2020;43(2):290-7.

26. Merli M, Lattanzi B, Aprile F. Sarcopenic obesity in fatty liver. Curr Opin Clin Nutr Metab Care. 2019;22(3):185-90.

27. Azzu V, Vacca M, Virtue S, Allison M, Vidal-Puig A. Adipose tissue-liver cross talk in the control of whole-body metabolism: implications in non-alcoholic fatty liver disease. Gastroenterology. 2020;158(7):1899-912.

28. Pedersen BK, Febbraio MA. Muscles, exercise and obesity: skeletal muscle as a secretory organ. Nat Rev Endocrinol. 2012;8(8):457-65.

29. Seldin MM, Peterson JM, Byerly MS, Wei Z, Wong GW. Myonectin (CTRP15), a novel myokine that links skeletal muscle to systemic lipid homeostasis. J Biol Chem. 2012;287(15):11968-80.

30. Pasiakos SM, Cao JJ, Margolis LM, Sauter ER, Whigham LD, McClung JP, et al. Effects of high-protein diets on fat-free mass and muscle protein synthesis following weight loss: a randomized controlled trial. FASEB J. 2013;27(9):3837-47.

31. Belizario JE, Fontes-Oliveira CC, Borges JP, Kashiabara JA, Vannier E. Skeletal muscle wasting and renewal: a pivotal role of myokine IL-6. Springerplus. 2016;5:619.

32. Fukushima Y, Kurose S, Shinno H, Thi Thu HC, Takao N, Tsutsumi H, et al. Effects of body weight reduction on serum irisin and metabolic parameters in obese subjects. Diabetes Metab J. 2016;40(5):386-95.

\section{Publisher's Note}

Springer Nature remains neutral with regard to jurisdictional claims in published maps and institutional affiliations.
Ready to submit your research? Choose BMC and benefit from:

- fast, convenient online submission

- thorough peer review by experienced researchers in your field

- rapid publication on acceptance

- support for research data, including large and complex data types

- gold Open Access which fosters wider collaboration and increased citations

- maximum visibility for your research: over 100M website views per year

At BMC, research is always in progress.

Learn more biomedcentral.com/submissions 